\title{
LA PENALIZACIÓN DE LAS RELACIONES SEXUALES ENTRE O CON ADOLESCENTES Y SU EFECTO EN EL EJERCICIO DE LOS DERECHOS SEXUALES Y REPRODUCTIVOS
}

\begin{abstract}
Rossina Guerrero ${ }^{1, a}$
RESUMEN

El artículo analiza los efectos en el Perú de la aprobación de la Ley 28704 en el año 2006, la cual determinó que toda relación sexual entre los 14 y menos de 18 años, así sea consentida, fuera considerada una violación sexual, la cual criminaliza la sexualidad adolescente y originar una situación de ilegalidad de los servicios de prevención en salud reproductiva. Esta decisión se dio sin considerar los marcos legales que reconocen y protegen los derechos sexuales y reproductivos, las necesidades de los adolescentes en el campo de su sexualidad, así como sus repercusiones en los problemas de salud pública. En el año 2012, esta Ley se declaró inconstitucional. El cumplimiento de esta sentencia debería permitir implementar y mejorar las políticas públicas de educación sexual y el acceso a servicios de salud de calidad; en busca de generar leyes más efectivas que protejan a los adolescentes de la violencia sexual.
\end{abstract}

Palabras clave: Derechos sexuales y reproductivos; Adolescente; Normas Legales, Perú (fuente: DeCS BIREME).

\section{THE CRIMINALIZATION OF SEXUAL RELATIONS BETWEEN OR WITH ADOLESCENTS AND ITS IMPACT ON THE EXERCISE OF THEIR SEXUAL AND REPRODUCTIVE RIGHTS}

\begin{abstract}
This article analyzes the impact of the decrete 28704 , issued in 2006 , on the Peruvian society. This Law states that all sexual relations between the age of 14 and an age younger than 18, whether consented or not, shall be considered rape, thus criminalizing adolescent sexuality and making preventive reproductive health services illegal. This decision was made disregarding the legal frameworks which recognize and protect sexual and reproductive rights, the needs of adolescents regarding sexuality, as well as its impact on public health issues. In 2012, this Law was declared unconstitutional. The enforcement of this sentence shall enable the implementation and improvement of public sexual education policies and the access to quality health care. An effort to create more effective laws to protect adolescents from sexual violence must be made.
\end{abstract}

Key words: Sexual and reproductive rights; Adolescent; Enacted Statutes, Peru (source: MeSH NLM).

\section{INTRODUCCIÓN}

Los efectos adversos que tuvo la aplicación de la Ley 28704 en la salud sexual y reproductiva de los adolescentes han sido reconocidos por diferentes sectores sociales y políticos en el Perú, desde el Estado, que incluyen al Ministerio de Salud, el Ministerio de la Mujer y Poblaciones Vulnerables, la Defensoría del Pueblo y el Congreso de la República y, por supuesto, la sociedad civil.

La aprobación de esta reforma legal no consideró los marcos legales tanto a nivel nacional como internacional, que habian permitido, con muchas deficiencias, mejorar el acceso a servicios de salud reproductiva a los adolescentes, en busca de superar, principalmente, las barreras de acceso a métodos anticonceptivos temporales, ello reflejado en la Norma Técnica de Planificación Familiar del año $2005^{(1)}$. Esta estipula el acceso a estos métodos, previa consejería, para adolescentes que presenten riesgo de embarazo no deseado, de contraer una ITS o VIH-SIDA, para quienes sean sexualmente activos/as, con antecedente de embarazo y para víctima de violación sexual.

La ley generó en los servicios de salud una situación más que irregular, con ello quienes más sufrieron sus efectos fueron los propios adolescentes a quienes puso en situaciones de mayor vulnerabilidad para su salud y vida, y los colocó en la situación de infractores o delincuentes ${ }^{(2)}$.

Centro de Promoción y Defensa de los Derechos Sexuales y Reproductivos (PROMSEX). Lima, Perú.

Licenciada en psicología

Recibido: 28-06-13 Aprobado: 04-09-13

Citar como: Guerrero R. La penalización de las relaciones sexuales entre o con adolescentes y su efecto en el ejercicio de los derechos sexuales y reproductivos. Rev Peru Med Exp Salud Publica. 2013;30(3):500-5. 
La aplicación de esta Ley afectó de manera adversa la implementación de políticas para mejorar y proteger la salud sexual y reproductiva de los adolescentes, un ejemplo de ello es que hasta la fecha, el Ministerio de Salud no logra aprobar el Plan Multisectorial de Prevención del Embarazo Adolescente, siendo la principal barrera la vigencia de esta Ley.

Luego de diferentes esfuerzos por visibilizar sus efectos adversos tanto desde la sociedad civil como desde diversos sectores del propio Estado, y llevar adelante esfuerzos por modificaciones desde el Congreso de la República, todas fallidas, finalmente el Tribunal Constitucional el año 2012, en su Sentencia 000082012-PI/TC declaró fundada la demanda interpuesta por 10609 ciudadanos y, con ello, la inconstitucionalidad del artículo $173 .^{\circ}$, inciso 3 del Código Penal, que penaliza las relaciones sexuales consentidas entre y con adolescentes de 14 hasta menos de 18 años de edad ${ }^{(3)}$.

La Sentencia del Tribunal Constitucional, deja acciones que deben ser cumplidas por el Congreso de la Republica, por el Poder Judicial y por los ministerios de salud y educación a fin que su cumplimiento sea una realidad y que se logre con compromiso político y designación de recursos para cumplir y mejorar las políticas públicas vigentes en materia de derechos sexuales y reproductivos de los adolescentes.

\section{AFECTACIÓN DE LOS DERECHOS HUMANOS EN EL CAMPO DE LA SEXUALIDAD Y REPRODUCCIÓN}

A partir del 6 de abril de 2006: Toda relación sexual que se desarrolle teniendo uno de los intervinientes (varón o mujer) menor de 18 años, pero con más de 14 es considerado como delito, aun cuando se preste el consentimiento expreso y no haya (violencia o grave amenaza) o engaño. Lo mismo ocurre cuando las relaciones sexuales se realizan entre adolescentes o jóvenes de edad semejante (entren los 14 y 18 años), por ejemplo, dentro de una relación afectiva-emocional. Aquí estaremos ante menores infractores $y$, pese a que no se abrirá proceso penal, los adolescentes serán sometidos a las reglas del Código de los Niños y Adolescentes.

A partir de ello, y teniendo como precedente la vigencia del artículo $30 .^{\circ}$ de la Ley General de Salud del año 1997, que señala:

Artículo $300^{\circ} .-$ El médico que brinda atención médica a una persona herida por arma blanca, herida de bala, accidente de tránsito o por causa de otro tipo de violencia que constituya delito perseguible de oficio o cuando existan indicios de aborto criminal, está obligado a poner el hecho en conocimiento de la autoridad competente.

Los médicos, estuvieron en la obligación de poner en conocimiento de la autoridad competente (la Fiscalía) a las supuestas víctimas de violación. Tal como lo refiere la Directiva 001-2007-DSDJL-MP-FN que establece la obligación de los responsables de los establecimientos de salud privados y públicos de comunicar a las fiscalías en el plazo máximo de 72 horas la atención o el ingreso a nosocomio de niñas y adolescentes menores de dieciocho años en estado de gestación. Se puede suponer que todas estas adolescentes y sus parejas fueron puestas a disposición de la Fiscalía de la Nación para inicio de la investigación penal $(4,5)$.

Como en toda relación humana, para que la información fluya durante un acto médico, se precisa de "confianza"; asimismo, se requiere establecer una relación que permita que la información sea lo más veraz posible. Por ello, un sistema de salud que no garantice la generación de confianza producirá actos médicos no fiables $y$, probablemente, no se logrará el objetivo de un beneficio en la salud de las personas/pacientes. Así, lejos de cumplir con el cometido de proteger la integridad de los adolescentes y de garantizar su desarrollo y su calidad de vida presente y futuro, se vulneró sus derechos sexuales y reproductivos.

La Corte Interamericana de Derechos Humanos ha tenido oportunidad de pronunciarse sobre el derecho de los médicos al secreto profesional en el caso De la Cruz Flores contra el estado peruano, en su Sentencia del año 2004. En torno a la obligación de denuncia respecto a posibles actos delictivos por parte de los médicos, la $\mathrm{CIDH}$ planteó las siguientes consideraciones:

a) Que la información que el médico obtiene en ejercicio de su profesión se encuentra privilegiada por el secreto profesional;

b) Que los médicos tienen un derecho y un deber de guardar confidencialidad sobre la información a la que tengan acceso en su condición de médicos;

c) Que las leyes nacionales deben ser modificadas en el sentido de proteger la confidencialidad de la información médica.

La aplicación de ambas Leyes establecieron que el "tamiz" del delito fueran los servicios de control del embarazo o parto. El embarazo se constituyó en la prueba más fehaciente de la actividad sexual y el mecanismo de detección del delito. Las adolescentes y/o sus parejas tenían que ser reportadas a la Fiscalía como víctimas de violación o como posibles violadoras/es. Ello trajo un serio problema en el sistema judicial, tal como señala 
Samuel Abad ${ }^{(6)}$, el Ministerio Público y el Poder Judicial han tenido que enfrentar este tipo de situaciones surgidas con motivo de la vigencia de la ley cuestionada. Este último, incluso, ha hecho uso del control difuso de reputar la norma como inconstitucional y además, ha elevado el tema a dos "acuerdos plenarios". Son pocos los casos públicos conocidos, pero ejemplo de ello son los vistos por la Segunda Sala Penal de la Corte Superior de Arequipa en los casos Tome Guillén, Paraguayo Quispe y Jihuallanca Arapa, la cual implicó la Ley.

Por otro lado, fue reconocido que también los servicios de provisión de métodos anticonceptivos se pusieron en riesgo, pues si el código tipifica como delito toda relación sexual de los adolescentes, sea consentida o no, ello convierte en ilegales las atenciones en salud sexual y reproductiva para los adolescentes. En este sentido, si estas no están orientadas a la abstinencia sexual, son ilegales, incluso los servicios de prevención del embarazo y de prevención de infecciones de transmisión sexual ${ }^{(7-9)}$.

Se puede comprender que esta reforma legal pretendía mejorar al marco de protección a las adolescentes ante una violación sexual, y que el contexto estaba marcado por numerosos casos de violación que se hicieron a menores de edad. Por ello, tuvo el respaldo de diferentes grupos políticos y sociales, que incluyeron organizaciones de la sociedad civil, quienes en ese momento no la valoraron como peligrosa, a costa de no considerar que los adolescentes tienen vida sexual activa, la cual también es consentida, según la ENDES continua del 2012 el 50\% de adolescentes tuvieron su primera relación sexual antes de los 15 años.

\section{LA CAPACIDAD DE LOS ADOLESCENTES DE TOMAR DECISIONES EN MATERIA SEXUAL}

El Comité de Derechos del Niño de las Naciones Unidas ha definido la adolescencia como: Un período caracterizado por rápidos cambios físicos, cognoscitivos y sociales, incluida la madurez sexual y reproductiva, la adquisición gradual de asumir comportamientos y funciones de adultos, que implica nuevas obligaciones y exigen nuevos conocimientos teóricos y prácticos.

La adolescencia es un período trascendental en el desarrollo de la sexualidad, se caracteriza por transformaciones corporales, entre las que la sexualidad emerge como parte del proceso de cambio biopsicosocial que influye profundamente en la vida presente y futura de la persona. Desde el punto de vista psicológico, la adolescencia es un período caracterizado por la evolución de los procesos psicológicos que implican cambios y crecimiento emocional, psicológico, social y mental, en el que las personas buscan y definen su propia identidad y se autoafirman como individuos, se apropian de valores y amplían su mundo de referencia social, más allá de su familia ${ }^{(10)}$.

Todo ello implica que en esta etapa, el ser humano deja de ser un niño, definido como una persona carente de madurez física y mental, y se va convirtiendo en un ser humano autónomo que define su propia personalidad y sus propias emociones, valores, su identidad en conjunto y, en consecuencia, se encuentra en capacidad de disfrutar de mayores espacios de autonomía, y es capaz de tomar decisiones propias y conscientes.

En consideración a ello, los ordenamientos jurídicos de diferentes países, incluyendo los de América Latina, han establecido edades para consentimiento sexual que oscilan entre los 14 y 15 años de edad, tal como se aprecia en la Tabla 1.

Lo señalado anteriormente da cuenta de la reconocida capacidad progresiva que tienen los adolescentes para tomar decisiones, lo cual, fue y sigue siendo escasamente comprendido y, a veces, negado. Ello llevó a generar una norma legal que no tuvo como base la evidencia científica, ni la de salud pública y un marco de derechos humanos. Aun más, generó una desarmonización legislativa en materia de derechos, ya que el Código Civil vigente reconoce la capacidad a los adolescentes desde los 14 años para actos relacionados con su sexualidad y la reproducción. Pueden a) reconocer a sus hijos; b) demandar los gastos de embarazo y el parto; c) demandar y ser parte en los procesos de reconocimiento judicial de filiación extramatrimonial, tenencia y alimentos de los hijos, y d) casarse desde los 16 años. En este último caso, todas las relaciones sexuales dentro del matrimonio configurarían delito de violación, lo cual carece de toda lógica ${ }^{(12-13)}$.

Tabla 1. Edades de consentimiento sexual reconocidos en normas legales

\begin{tabular}{lc}
\hline \multicolumn{1}{c}{ País } & Edad de consentimiento sexual \\
\hline Bolivia & Pubertad \\
Chile & 14 \\
Ecuador & 14 \\
Colombia & 14 \\
Costa Rica & 15 \\
Argentina & 13 \\
Brasil & 14 \\
Uruguay & 15 \\
Panamá & 14 \\
Paraguay & 14 \\
\hline Tomado y Adaptado de: Salas Jorge (11).
\end{tabular}




\section{LAS CIFRAS DAN CUENTA DE LOS EFECTOS ADVERSOS}

Las políticas públicas destinadas a mejorar la salud sexual y reproductiva de este grupo de ciudadanos, que ya enfrentaban escasa prioridad política y barreras sociales y culturales en autoridades y proveedores de salud, entraron en "suspensión" o, bajo un enfoque de derechos humanos, fueron criminalizadas.

El Ministerio de Salud fue uno de los primeros sectores del Estado en explicitar su preocupación por los efectos negativos de esta reforma legal, dio cuenta que la fecundidad en las adolescentes se volvió a incrementar desde el año 2007, que la mortalidad materna adolescente aumentó, aunque la mortalidad materna general había disminuido entre el año 2006 y 2008, que disminuyó la demanda de los adolescentes a los servicios ofertados por el MINSA, pese a que la población adolescente ha aumentado y que la cobertura de filiación a este grupo poblacional es mayor que años anteriores y que un porcentaje significativo de las usuarias insatisfechas en planificación familiar eran adolescentes.

En el año 1996, de acuerdo con la ENDES, la fecundidad adolescente era de 13\%, y dieciséis años más tarde, es decir, en el 2012, la fecundidad adolescente fue de $13,2 \%$. Sin embargo, los promedios ocultan brechas e inequidades, la ENDES 2012 reporta cifras preocupantes de embarazo adolescente en algunas regiones del país, las más notables son Loreto con $32,2 \%$; San Martin con $27,2 \%$; Tumbes con $27,0 \%$, y Ucayali con $26,5 \%$. Señala también que la mayor prevalencia de embarazo adolescente se encuentra en mujeres sin educación (56,7\%); en las residentes de la selva $(27,5 \%)$; entre las que son pobres $(26,1 \%)$, y entre las que provienen del área rural $(21,5 \%)$. La ENDES 2011 señala además que $71,1 \%$ de las mujeres menores de 20 años tuvieron embarazos no deseados, pues los querían más tarde $(63,2 \%)$ o simplemente no los querían $(7,9 \%)^{(14-16)}$.

Sin duda, ello coloca al Perú en una tarea impostergable de implementar políticas efectivas que permitan prevenir el embarazo adolescente, ya que los impactos negativos en lo social y económico han sido vastamente reconocidos. Las adolescentes embarazadas son marginadas en los espacios educativos, familiares y laborales y aún persiste la tendencia a expulsarlas de las escuelas, o caer en otras formas de discriminación ${ }^{(17) .}$

Por otro lado, el uso de métodos anticonceptivos también se afectó; las barreras sociales y culturales unidas a los fenómenos de desabastecimiento de métodos anticonceptivos y a la barrera legal estaría explicando

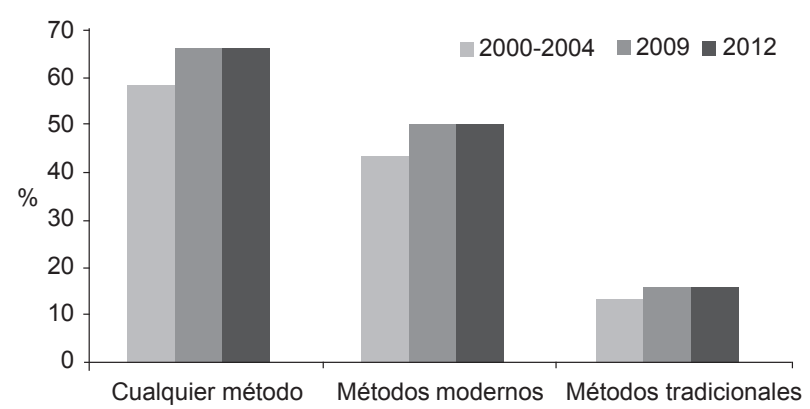

Figura 1. Porcentaje de uso de métodos anticonceptivos en adolescentes unidas de 15 a 19 años en Perú

que en más de 10 años la tasa de embarazo adolescente se mantenga invariable junto con el bajo nivel de uso de métodos anticonceptivos modernos, donde no más del $50 \%$ de adolescentes unidas usa actualmente uno de ellos (Figura 1). Ello no solo es grave en términos de la prevención del embarazo no deseado, sino también del aborto inseguro y la muerte materna, que tienen como principal estrategia el aseguramiento y acceso a métodos anticonceptivos modernos.

La Mesa de Concertación para la Lucha contra la Pobreza (MCLCP), no fue ajena a reconocer esta grave situación. En el 2012, señaló que la Ley 28704 no contribuía a proteger los derechos sexuales y reproductivos de los adolescentes, sino que ha impactado negativamente en el acceso a la salud sexual y reproductiva, en el control prenatal y la atención del parto, alejando a los adolescentes por temores a las sanciones legales ${ }^{(18)}$.

La MCLCP alertó que en el 2010 la muerte materna en adolescentes entre los 10 y 19 años era del $12 \%$ respecto al total de muertes; dentro de las causas de muerte indirecta, el suicidio aparecía con un preocupante $39 \%$; asimismo, reportaba un incremento de la incidencia de abortos en adolescentes del 17,6\% en el 2006 al 20,2\% en el 2008. De acuerdo con la Dirección General de Epidemiologia del Ministerio de Salud, en el año 2012, del total de muertes maternas notificadas en el país (428 casos), el $9,6 \%$ se produjo en adolescentes entre los 12 y 17 años ${ }^{(19)}$.

Las cifras terminan de dar cuenta de la situación límite en la cual muchas adolescentes vienen afrontando su sexualidad, por una medida legislativa que se constituyó en vulneradora de sus derechos sexuales y reproductivos, $y$ ha puesto en grave riesgo el aprovechamiento de las mejores capacidades de ciudadanos para aportar al desarrollo de sus comunidades y del país en general. 


\section{EL TRIBUNAL CONSTITUCIONAL Y EL DERECHO A DECIDIR}

El 2008, la Corte Suprema de Justicia de la República, aprobó su Acuerdo Plenario 4-2008/CJ-116, señalando que cuando la víctima es mayor de 14 y menor de 18 años, y existe consentimiento de su parte, el agente quedará exento de responsabilidad penal. Estableciendo que las relaciones sexuales consentidas no son punibles, es decir, no constituyen delito ${ }^{(20)}$.

Esta respuesta acertada del Poder Judicial, permitió que a partir de la fecha, muchos adolescentes no tuvieran que enfrentar procesos judiciales, pero no impedía que los servicios de salud sigan notificando ante la Fiscalía sus atenciones.

Es por ello que se necesitaba la reforma legal, luego de intentos sin éxito en el Congreso de la República para lograrlo, el año 2012, el Tribunal Constitucional mediante su Sentencia 00008-2012-PI/TC declaró fundada la demanda interpuesta de 10609 ciudadanos $\mathrm{y}$, con ello, la inconstitucionalidad del artículo $173 .{ }^{\circ}$ inciso 3 del Código Penal, que penaliza las relaciones sexuales consentidas entre y con adolescentes de 14 hasta menos de 18 años de edad.

Esta sentencia reconoció a los adolescentes entre 14 y 18 años de edad la titularidad del derecho a la libertad sexual, que como señaló, forma parte de la vida estrictamente privada de toda persona, en lo que refiere a la toma de decisiones sobre su sexualidad. Asimismo, consideró que el Estado es el llamado a garantizar que los adolescentes ejerzan su derecho a la información, lo que les permita disfrutar de su sexualidad, afirmando que uno de los principales obligados a garantizar los derechos a la información, a la salud y la intimidad es el Estado.

En este sentido, el Estado tiene el deber de no interferir con acciones que restrinjan el ejercicio de ese derecho, pero al mismo tiempo tiene la obligación de crear reales posibilidades de acceso a servicios de salud de calidad e información necesaria sobre su sexualidad sin discriminación.

\section{REFLEXIONES FINALES}

Este debate público puso en evidencia un sinnúmero de estereotipos sobre la sexualidad de los adolescentes, centrados en la negativa de aceptarlos como seres sexuados, y con derechos; y en una visión negativa de la experiencia sexual.

El Tribunal Constitucional zanjó este debate desde lo jurídico, y reafirmó a los adolescentes entre los 14 y 18 años no solo como objeto de derecho, sino como sujetos de derecho y con capacidad para decidir sobre su sexualidad. Este ha sido sin duda un avance importante; sin embargo, queda un largo tramo importante por trabajar en lo relacionado a los cuestionamientos vigentes por tomadores de decisión, profesionales de salud y otros agentes, respecto a la sexualidad adolescente, lo cual seguirá afectando la implementación de políticas públicas.

El Plan Nacional de Acción por la Infancia y Adolescencia 2012-2021 y el Plan Nacional de Población 2010-2014, políticas públicas vigentes, reconocen de manera expresa la necesidad de proteger y garantizar la salud y los derechos sexuales y reproductivos de los adolescentes, incluyendo metas e indicadores emblemáticos que deben cumplirse en materia de prevención embarazo adolescente, del VHI/ SIDA y violencia sexual y acceso a servicios de salud.

Finalmente, señalar que nunca debió hacer que los adolescentes se convirtieran en delincuentes. Si se desea erradicar y protegerlos de la violencia sexual, se deben emprender medidas que impliquen mayor inversión en políticas de prevención y de acceso a la justicia para las víctimas, incluyendo una educación sexual que genere nuevas relaciones de poder entre los sexos con el compromiso eficiente de los altos niveles de decisión política.

Fuentes de financiamiento: autofinanciado.

Conflictos de interés: el autor declara no tener conflictos de interés.

\section{REFERENCIAS BIBLIOGRÁFICAS}

1. Perú, Ministerio de Salud. Norma Técnica de Planificación Familiar. NT $\mathrm{N}^{\circ}$ 032-MINSA/DGSP-V.01. Lima; MINSA;. 2005.

2. Castillo J. La muerte de la sexualidad en los adolescentes. La Ley 28704 y la irresponsabilidad del legislador. Gaceta Jurídica. 2006:14.
3. Nagle J, Chávez S. De la protección a la amenaza: consecuencias de una ley que ignora los derechos sexuales y reproductivos de las y los adolescentes. El caso de la modificatoria del Código Penal, Ley 28704. Lima: Promsex; 2007.

4. Gianella G. El sentido y la importancia del secreto profesional desde la medicina. En: Gamarra R, Uceda R, Fianella G. Secreto profesional: análisis y perspectiva de la medicina, el periodismo y el derecho. Lima: Promsex; 2011. p. 65-98.

5. Loayza Tamayo C, Marín Sandoval Y.E Derecho de las médicas y los médicos al secreto profesional en la jurisprudencia 
de la Corte Interamericana de Derechos Humanos. Lima: Promsex; 2010.

6. Abad, S. Informe sobre la inconstitucionalidad del inciso 3 del artículo 173 del Código Penal Peruano. Lima: Promsex; 2011.

7. Ramírez, B. Protección contra la violencia sexual hacia adolescentes y respeto a sus derechos fundamentales. A propósito del artículo 173.3 del Código Penal y la RTC Exp. N. ${ }^{\circ} 00018$ 2011-PI/TC. Gaceta Constitucional. 2012; $49: 22-32$.

8. Naciones Unidas, Comité de Derechos del Niño. Observación General N. ${ }^{\circ}$ 14, la Salud y el desarrollo de los adolescentes en el contexto de la Convención sobre los Derechos del Niño. CRC/GC/2003/4 [Internet]. Geneva: United Nations; 2003 [citado el 12 de febrero del 2013]. Disponible en: http://www.unhchr. ch/tbs/doc.nsf/(Symbol)/CRC. GC.2003.4.Sp?Opendocument

9. Contreras J, Hakkert R. La sexualidad y la formación de uniones. En: Guzmán J, Hakker R, Contreras J, Falconier de Moyano M. Diagnósticos sobre salud sexual y reproductiva de adolescentes en América Latina y el Caribe. México: UNFPA; 2001. p. 57-104.

10. Chávez A, Guerreo R, Espinoza J, Ayzanoa G. Concepciones y percepciones sobre los derechos sexuales y reproductivos en la adolescencia. Lima. PROMSEX; 2007.

11. Salas Arenas JL. Derecho a la libertad sexual de los y las adolescentes [Internet]. II Congreso Latinoamericano Jurídico sobre Derechos Reproductivos. San José, Costa Rica, 28, 29 y 30 de noviembre de 2011 [citado el 28 de junio del 2013]. Disponible en: http:// segundo.congresoderechosreproductivos.com/files/presentaciones/PONENCIAJORGESALASARENAS. pdf

12. Ramírez Huaroto B. Reforma legislativa para despenalizar las relaciones sexuales consentidas en adolescentes. Lima: Promsex; 2011.

13. Perú, Ministerio de Salud. Análisis de situación de salud de las y los adolescentes: ubicándolos y ubicándonos. Lima: MINSA; 2009.

14. Perú, Instituto Nacional de Estadística e Informática. Perú: Situación social de las madres adolescentes, 2007. Lima INEI; 2010.

15. Perú, Instituto Nacional de Estadística e Informática. Encuesta Demográfica y de Salud Familiar - ENDES 1996. Informe Principal. Lima: INEI; 1997.

16. Perú, Instituto Nacional de Estadística e Informática. Encuesta Demográfica y de Salud Familiar - ENDES Continua, 2012. Informe Principal. Lima: INEI; 2013.
17. CEPAL, UNICEF. Maternidad adolescente en América Latina y el Caribe. Tendencias, problemas y desafíos. Desafíos. 2007;(4).

18. Mesa de Concertación de Lucha contra la Pobreza; Grupo de Trabajo para la Prevención del Embarazo Adolescente. Prevención del embarazo adolescente en el Perú. Por una mayor calidad de vida de las y los adolescentes. Lima: INPPARES; 2012.

19. Perú, Ministerio de Salud, Dirección General de Epidemiología. Sistema Nacional de Vigilancia Epidemiológica 2012 [Internet]. Lima: Dirección de Epidemiología; c2013 [citado el 27 de junio de 2012]. Disponible en: http:// www.dge.gob.pe/salasit.php

20. Perú, Corte Suprema de Justicia. IV Pleno Jurisdiccional de las Salas Penales, Permanente, Transitorias y Especial. Acuerdo Plenario N. ${ }^{\circ}$ 4- 2008/CJ116. Aplicación del artículo $173^{\circ}, 3$ del Código Penal. Delito de violación sexual a menor de edad. Lima: Promsex, Movimiento Manuela Ramos; 2009.

Correspondencia: Rossina Guerrero

Dirección: Av. José Pardo 601. Oficina 604 Miraflores Lima - Perú

Teléfono: $51-1-4478668$

Correo electrónico: rossina@promdsr.org

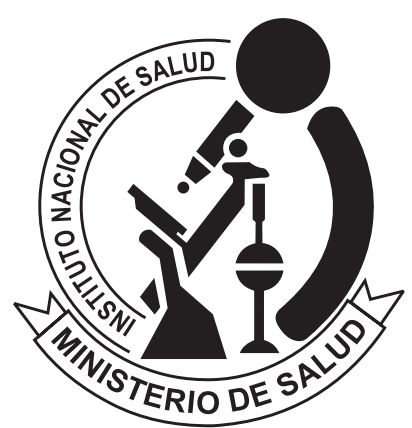

Investigar para proteger la salud
REVISTA PERUANA DE MEDICINA EXPERIMENTAL Y SALUD PÚBLICA CUMPLIENDO SUS METAS $Y$ PROYECTÁNDOSE AL FUTURO

\author{
Visite los contenidos de la revista en: \\ www.ins.gob.pe/rpmesp
}

